\title{
Newcomers meet the intracluster medium in the Coma cluster
}

\author{
Bianca M. Poggianti ${ }^{1}$, T. J. Bridges ${ }^{2}$, M. Yagi ${ }^{3}$, Y. Komiyama ${ }^{4}$, \\ D. Carter ${ }^{5}$, B. Mobasher ${ }^{6}$, S. Okamura ${ }^{7}$ and N. Kashikawa ${ }^{3}$ \\ ${ }^{1}$ INAF-Osservatorio Astronomico di Padova, Italy \\ 2 Anglo-Australian Observatory, Australia \\ ${ }^{3}$ National Astronomical Observatory, Mitaka, Tokyo, Japan \\ ${ }^{4}$ Subaru Telescope, Hilo, HI, USA \\ ${ }^{5}$ Liverpool John Moores University, Birkenhead, Wirral, UK \\ ${ }^{6}$ Space Telescope Science Institute, Baltimore, USA \\ ${ }^{7}$ Department of Astronomy, University of Tokyo, Japan
}

\begin{abstract}
A main topic at this meeting is how galaxies are affected when they enter for the first time the cluster environment from the outskirts. Most of the times we are forced to infer the environmental effects indirectly, relying on systematic variations of galaxy properties with environment, but there aren't many examples of direct observations able to unveil ongoing transformations, and the corresponding mechanism producing it. We present a case in which it is possible to identify the cluster environment, and in particular the intracluster medium and the recent infall history of galaxies onto the cluster, as the cause for a recent, abrupt change in the evolutionary history of galaxies.
\end{abstract}

\section{Post-starburst galaxies and substructure in Coma}

This study is based on a photometric and spectroscopic survey of galaxies in the Coma cluster (Mobasher et al. 2001), which is distinctive from other surveys in three ways: for the large galaxy magnitude range covered (almost 7 mag, down to $M_{B} \sim-14$ ), for the large area surveyed (two regions, towards the cluster center and to the SouthWest, $\sim 1 \times 1.5 \mathrm{Mpc}$ each), and for being a simply magnitude limited sample, with no morphological selection criteria adopted for spectroscopy. In this survey we have found that a significant fraction $(\sim 10 \%)$ of the cluster dwarf galaxy population at $M_{V}>-18.5$ has post-starburst/post-starforming spectra (Poggianti et al. 2004, hereafter P04). This type of spectrum ("k+a", or "E+A") indicates a galaxy with no current star formation activity which was forming stars at a vigorous rate in the recent past (last $1.5 \mathrm{Gyr}$ ). In the $B-R$ color-magnitude diagram, a group of blue and a group of red $\mathrm{k}+$ a galaxies can be easily distinguished in Coma. The average $\mathrm{EW}(\mathrm{H} \delta)$ of the blue group is significantly stronger than that of the red group. The blue, strong $\mathrm{k}+\mathrm{a}$ 's most likely correspond to "young" k+a's (observed soon after the termination of star formation, < $300 \mathrm{Myr}$ ) and the red, weaker k+a's are "old" ones (observed at a later stage of the evolution, 0.5-1.5 Gyr).

A suggestive clue about the possible physical mechanism responsible for the $\mathrm{k}+\mathrm{a}$ spectra comes from the recent X-ray mosaic observations of Coma obtained with $X M M$ Newton. Coma has two central dominant galaxies, NGC 4874 (a cD galaxy) and NGC4889 (a very bright elliptical), and another cD galaxy, NGC4839, that dominates a substructure South-West of the center (Fig. 1). Neumann et al. (2003) have identified and 


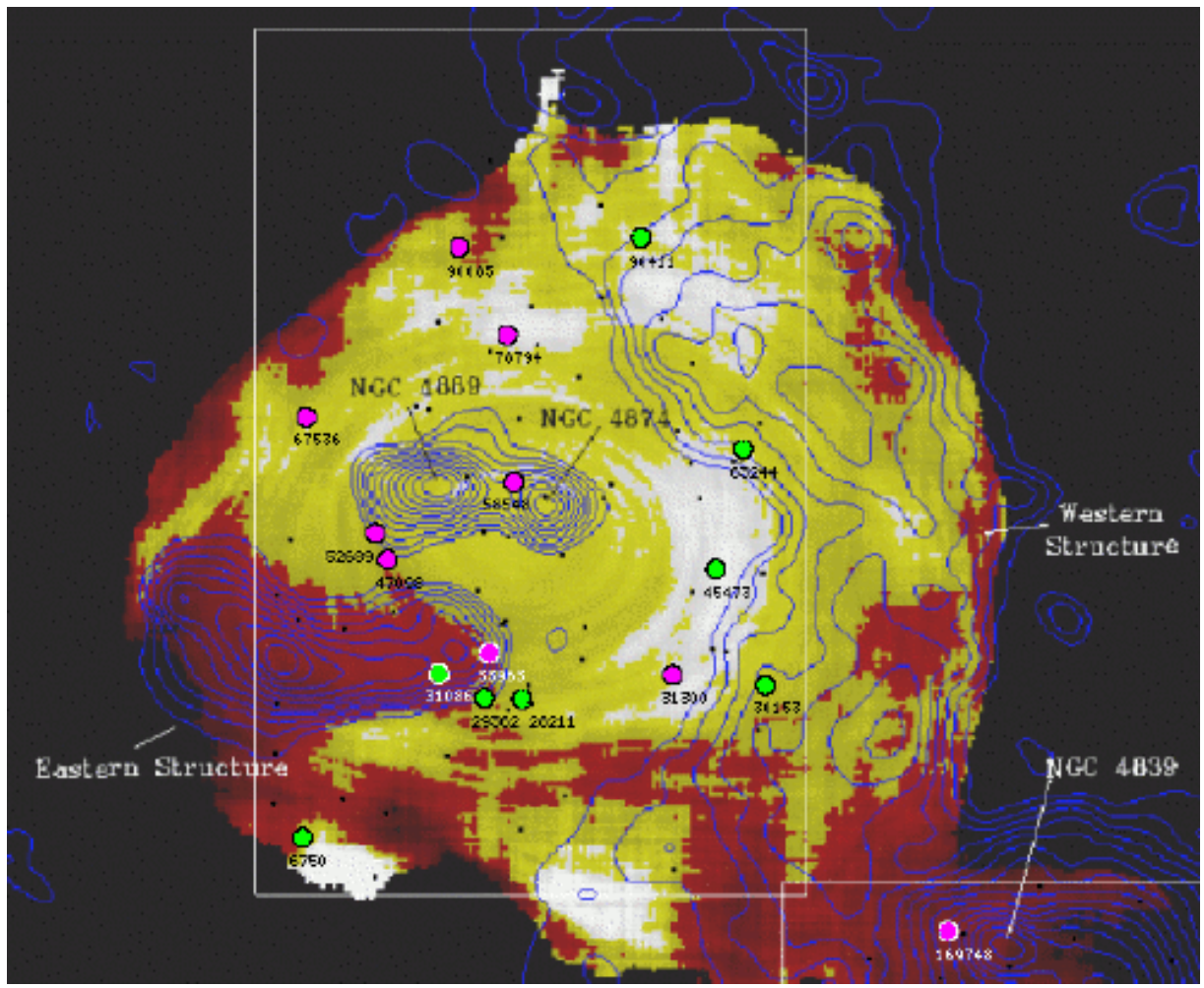

Figure 1. N.B. This plot is best viewed in color, see Poggianti et al. 2004. Position of $\mathrm{k}+\mathrm{a}$ galaxies with respect to X-ray substructure and X-ray temperature map. Only the central field of Coma is shown here, see P04 for a full map. Strong-lined k+a's with $\mathrm{EW}(\mathrm{H} \delta)>5$ $\AA$ are shown as light-coloured large dots, while weaker $\mathrm{k}+\mathrm{a}$ 's are plotted as darker large dots. Tiny black dots are dwarf Coma members with velocities $>7200 \mathrm{~km} \mathrm{~s}^{-1}$. X-ray residuals from Neumann et al. (2003) are plotted as contours and clearly identify two substructures (Western and Eastern substructures), in addition to the NGC4839 peak in the South-West and the excess of emission towards the two central galaxies (NGC4874 and NGC4889). The lowest contour and the step width between two contours are each $5 \sigma$. The hardness ratio image $(2-5 \mathrm{keV} / 0.5-2 \mathrm{keV}$, Neumann et al. 2003) is also shown. Darker regions correspond to temperatures below $8 \mathrm{keV}$, intermediate-grey regions to $k T>8 \mathrm{keV}$ and light-coloured regions to $k T>10 \mathrm{keV}$. The rectangles show the limits of the two fields of our photometric and spectroscopic survey (Coma1 towards the cluster center and Coma3 in the South-West). Each rectangle is about 1 by 1.5 Mpc.

discussed X-ray substructure by fitting a smooth profile and subtracting it from the data. The residuals reveal several structures, that are shown as contours in Fig. 1: besides the well known NGC4839 South-West group, Neumann et al. find a large residual to the West of the cluster centre ("Western structure" in Fig. 1) elongated along the North-South direction, and a filament-like structure South-East of the centre ("Eastern structure" in Fig. 1), elongated along the East-West direction. The temperature map shown in Fig. 1 sheds further light on the accretion history of Coma. Neumann et al. conclude that the region of high temperature observed between the Western structure and the Coma center is caused by the infall of this structure, either via compression or via shock waves. These authors consider the two maxima in the western structure to be likely the result of the disruption of a galaxy group during its infall, instead of two galaxy groups falling at the same time. In contrast, the South-Eastern structure is cooler than the mean cluster 
temperature and is associated with a low-mass galaxy group dominated by two large galaxies, NGC4911 and NGC4921. Based on the filamentary form of this structure, the same authors conclude it is observed during the infall process while being affected by ram pressure stripping close to the cluster centre.

The coincidence of the position of the strongest $\mathrm{k}+\mathrm{a}$ galaxies and the $\mathrm{X}$-ray structures is striking. Four $\mathrm{k}+\mathrm{a}$ 's with $\mathrm{EW}(\mathrm{H} \delta)>5 \AA$ (light large dots in Fig. 1) trace the edge of the Western structure towards the Coma centre. Another three are associated with the Eastern structure, all at its western boundary. Thus, young post-starbursts are distributed close to the edge of infalling substructures. In the case of the Western substructure this edge is the infalling front, while for the Eastern substructure it is unclear whether the group is moving to the West, as suggested by the appearance of the X-ray residuals, or to the East, as suggested by the positions of NGC4911 and NGC4921 (Neumann et al. 2003). Overall, this strongly suggests that the $\mathrm{k}+\mathrm{a}$ spectra, i.e. the truncation of the star formation activity in these galaxies and possibly the previous starburst, could be the result of an interaction with the hot intracluster medium (ICM).

In contrast, the location of the red $\mathrm{k}+\mathrm{a}$ galaxies in Fig. 1 does not appear to be correlated with the X-ray residuals. The red $\mathrm{k}+\mathrm{a}$ phase has a timescale that is comparable to the core crossing time in a cluster like Coma, and any signature of the link between the truncation of star formation and the location within a substructure is thus erased in the older $\mathrm{k}+\mathrm{a}$ 's, while it is still detectable in the youngest subsample of blue $\mathrm{k}+\mathrm{a}$ 's.

It is instructive to note that looking for a spatial segregation in the location of galaxies on the sky would not allow to establish a correlation between the star formation history of the $\mathrm{k}+\mathrm{a}$ galaxies and the substructure: the link with the dynamical history of Coma appears evident only once a detailed X-ray map reveals the complicated structure in the hot intracluster gas.

The blue $\mathrm{k}+\mathrm{a}$ 's do show, however, a radial velocity distribution that is significantly different from that of the red $\mathrm{k}+\mathrm{a}$ 's and the global Coma dwarf population. Their mean radial velocity is $8120 \pm 709 \mathrm{~km} \mathrm{~s}^{-1}$, with all but one at $v>7200 \mathrm{~km} \mathrm{~s}^{-1}$. In contrast, both the red $\mathrm{k}+\mathrm{a}$ 's and all faint galaxies with passive spectra have much lower mean velocities: $6992 \pm 761$ and $6854 \pm 244 \mathrm{~km} \mathrm{~s}^{-1}$, respectively.

\section{Implications}

Two main conclusions can be drawn from the analysis of the Coma $\mathrm{k}+\mathrm{a}$ galaxies.

a) The relation between $k+a$ galaxies and substructure within the Coma cluster. The position of faint post-starburst galaxies in Coma relative to X-ray substructure in the cluster strongly suggests that the interruption of the star formation activity in these galaxies is a cluster-related phenomenon, most likely due to the impact with the intracluster medium of recently in-fallen galaxy groups. Moreover, the physical process that caused the halting of the star formation must have acted on a short timescale: such timescale must be significantly shorter than $1 \mathrm{Gyr}$, the timescale for the visibility of the $\mathrm{k}+\mathrm{a}$ signature in the spectra, for a $\mathrm{k}+\mathrm{a}$ spectrum to be produced.

b) Comparing the properties of $k+a$ galaxies in Coma and in clusters at $z=0.4-0.5$ : the downsizing effect.

Numerous spectroscopic surveys of galaxies in distant clusters have found significant populations of luminous k+a galaxies (see for example Couch \& Sharples 1987, Abraham et al. 1996, Dressler \& Gunn 1992, Fisher et al. 1998, Dressler et al. 1999, Tran et al. 2003, but see also Balogh et al. 1999). However, $\mathrm{k}+\mathrm{a}$ galaxies as luminous as those in distant clusters $\left(M_{V} \leqslant-20\right)$ are absent in Coma, where $\mathrm{k}+\mathrm{a}$ 's are detected at magnitudes typically fainter than $M_{V} \sim-18$ (P04). 
Post-starburst galaxies in Coma are thus much fainter than those observed in distant clusters. $\mathrm{K}+\mathrm{a}$ spectra in clusters appear to be a luminous phenomenon at $z=0.5$ and a faint one at $z=0$. In Coma we are observing late-type star-forming galaxies becoming dwarf spheroidals, while the descendants of $\mathrm{k}+\mathrm{a}$ 's at high redshift will be among the most massive early-type galaxies today (see also Tran et al. 2003, and these proceedings). The observed evolution of the maximum luminosity of $k+a$ galaxies most probably reflects a change in the galaxy populations falling into clusters, and provides further evidence of a "downsizing effect": going to lower redshift, the maximum luminosity/mass of galaxies with significant star formation activity progressively decreases, in all environments, and active star formation in low mass galaxies seems to be more protracted on average than in massive galaxies.

\section{Related (mostly open) questions}

1. Based on the strength of the lines, most of the blue $\mathrm{k}+\mathrm{a}$ galaxies in Coma are inequivocally post-starburst systems, in which a starburst preceded the halting of the star formation. Was the starburst triggered by the impact with the intracluster medium? Based on timescale arguments, it is possible that the interaction with the ICM produces both the starburst and the subsequent halting of the star formation. Low-mass starforming galaxies, however, often have a burst-like, non-regular star formation history, and thus the possibility that the starburst could be an intrinsic non-environmentally related phenomenon cannot be excluded.

2. Could the termination of the star formation activity in these galaxies be due to "strangulation"? The $\mathrm{H} \delta$ strength of the blue $\mathrm{k}+\mathrm{a}$ galaxies implies that star formation was truncated in these galaxies on a short timescale, i.e. short compared to the $\mathrm{k}+\mathrm{a}$ timescale of 1-1.5 Gyr. A slowly declining star formation activity such as that envisaged if galaxies simply lost their gas halo reservoir when becoming part of a group ("strangulation", e.g. Bower \& Balogh 2003) is not able to produce such strong Balmer lines.

3. Do $k+a$ galaxies in distant clusters originate from the same physical process (the interaction with the ICM) that produced $k+a$ galaxies in Coma? This is yet unknown, and needs to be uncovered by studies of distant clusters (e.g. see Dressler et al. these proceedings).

4. Are there $k+a$ dwarf galaxies in distant clusters? So far, spectroscopy of distant cluster galaxies has only been obtained for relatively bright galaxies, thus it is unknown how many faint $\mathrm{k}+\mathrm{a}$ 's exist in clusters at high- $z$, and whether the luminous ones that have been observed are only the "tip of the iceberg".

5. Are field $k+$ a galaxies at low redshift dwarf galaxies too? Giant, massive $\mathrm{k}+\mathrm{a}$ galaxies are known in the field in the local Universe (e.g. Yang et al. 2004). Most likely field $\mathrm{k}+\mathrm{a}$ 's originate from a different mechanism (such as galaxy-galaxy mergers, Zabludoff et al. 1996) that has the same final effect of the dense environment: terminate the star formation activity.

6. In hierarchical models, small structures form first, and as a consequence low mass galaxies can be expected to have stellar populations on average older than more massive galaxies. Is the result presented here thus inconsistent with the predictions of semi-analytic modeling within a hierarchical scenario? This depends on the prescriptions adopted for the star formation recipe. Some recent models (e.g. De Lucia et al. in preparation) assume a star formation efficiency that increases with the halo mass, and thus obtain a trend of increasing age with galaxy mass for elliptical galaxies. 
7. And finally, what is the origin of the downsizing effect, i.e. of the fact that star formation in low mass galaxies appears to be more protracted on average than in massive galaxies? Most likely, since this phenomenon is known both in clusters (e.g. Smail et al. 1998, Kodama \& Bower 2001, De Lucia et al. 2004) and in the field (e.g. Cowie et al. 1996, Kauffmann et al. 2003), the mass dependence of the star formation history we observe in clusters simply reflects the evolution of the galaxy populations falling in from the field and it is intrinsic to the galaxy itself (e.g. related to its mass and due to the combination of gravity and self-regulating star formation, see Chiosi \& Carraro 2002), and not an environmental effect.

\section{References}

Bower, R.G. \& Balogh, M.L. 2003 in Carnegie Observatories Astrophysics Series, Vol.3: Clusters of Galaxies: Probes of Cosmological Structure and Galaxy Evolution eds. J.S. Mulchaey, A. Dressler and A. Oemler (Cambridge: Cambridge University Press), http://www.ociw.edu/ociw/symposia/series/symposium3/proceedings.html.

Chiosi, C., Carraro, G. 2002 MNRAS 335335.

Cowie, L.L., Songaila, A., Hu, E.M., Cohen, J.G. 1996 AnJ 112839.

De Lucia, G., and the EDisCS collaboration 2004 submitted (see also De Lucia et al. these proceedings).

Kauffmann, G., Heckman, T.M., White, S.D.M. et al. 2003 MNRAS 34154.

Kodama, T., Bower, R.G. 2001 MNRAS 32118.

Mobasher, B., Bridges, T. J., Carter, D., Poggianti, B. M., et al. 2001 ApJS 137279.

Poggianti, B.M., Bridges, T.J., Komiyama, Y., Yagi, M., Carter, D., Mobasher, B., Okamura, S., Kashikawa, N. 2004 ApJ 601197.

Smail, I., Edge, A.C., Ellis, R. S., Blandford, R.D. 1998 MNRAS 293124.

Tran, K.-V.H., Franx, M., Illingworth, G., Kelson, D.D., van Dokkum, P. 2003 ApJ 599865.

Yang, Y., Zabludoff, A.I., Zaritski, D., Lauer, T.R., Mihos, J.C. 2004 ApJ, in press (astro$\mathrm{ph} / 0402062)$.

Zabludoff, A. I., Zaritsky, D., Lin, H., Tucker, D., Hashimoto, Y., Shectman, S.A., Oemler, A., Kirshner, R.P. 1996 ApJ 466104. 